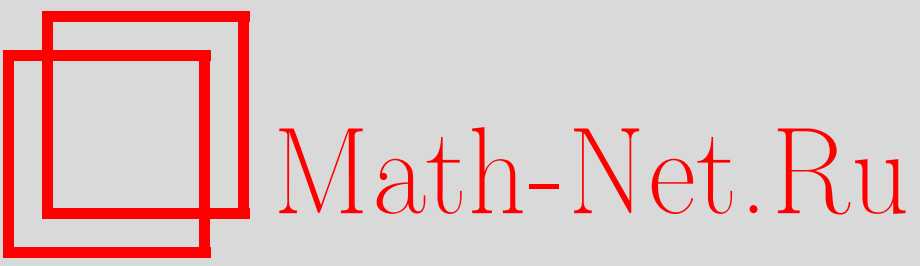

Е. Е. Скурихин, Вялые пучки на предпучках множеств, УМН, 1998, том 53, выпуск 6, 263-264

DOI: https://doi.org/10.4213/rm108

Использование Общероссийского математического портала Math-Net.Ru подразумевает, что вы прочитали и согласны с пользовательским соглашением

http: //www.mathnet.ru/rus/agreement

Параметры загрузки:

IP : 54.84 .234 .179

26 апреля 2023 г., 13:06:51 


\title{
ВЯЛЫЕ ПУЧКИ НА ПРЕДПУЧКАХ МНОЖЕСТВ
}

\author{
Е. Е. Скурихин
}

Как известно, вялые пучки работают на топологических пространствах с соверщенно разными топологическими свойствами. Свойства вялости используются при изучении производных функторов обратного предела $\lim ^{n}$. При разработке этальной теории также выделялись различные классы ацикличных пучков с тем же названием.

В данной работе рассматриваются вялые пучки на предпучках множеств, заданных на частично упорядоченном множестве (ч.у.м.) $K$, снабженном топологией Гротендика $\tau$. Если $K \subset O X$ $(O X$ решетка открытых подмножеств топологического пространства $X)$, то частньми случаями получающихся результатов являются, в зависимости от $\tau$, основные факты относительно вялых [1] и вялых нормальных пучков [2]. Во всех случаях получаемые резултаты относятся к функтору $\lim$ и его производным функторам $\lim _{\tau}^{(n)}$, совпадающим с $\lim (n)$, если $\tau$ дискретная топология Гротендика.

Напомним некоторые обозначения, относящиеся к предпучкам множеств (см. [3]-[6]). Пусть $(K, \tau)$ - сайт (т.е. категория $K$ и топология Гротендика $\tau$ на ней), $\widehat{K}$ - категория предпучков множеств на $K$. Для $D \in \mathrm{Ob}(\widehat{K})$ через $K_{D}$ и $K_{D, \tau}$ соответственно обозначаются множества всех и всех $\tau$-замкнутых подпредпучков $D$ (если $A \in K_{D}$, то $s \in[A]_{\tau}(k) \Leftrightarrow s \in D(k)$ и $\exists\left\{f_{i}: k_{i} \rightarrow\right.$ $k \mid i \in I\} \in \tau(k): s f_{i} \in A\left(k_{i}\right) ; A-\tau$-замкнут в $D$, если $\left.A=[A]_{\tau}\right)$. Отображения $j: K \rightarrow K_{1}$ и $j_{\tau}: K \rightarrow K_{1, \tau}$, где 1 - пучок на $K$, для которого множество $1(k)$ одноэлементное для всех $k$, определяются так: $j(k)(l)$ одноэлементно, если $\operatorname{Hom}(l, k) \neq \varnothing$, и пусто в противном случае; $j_{\tau}(k)=[j(k)]_{\tau}$.

ОПРеДЕЛЕнИЕ 1 . Пусть $\mathscr{U}$ - некоторая совокупность пар $(D, B)$ предпучков множеств на $K$, таких, что $B \subset D$. Назовем $\tau$-пучок $\mathscr{A} \mathscr{U}$-вялым, если $\forall(D, B) \in \mathscr{U}$ отображение ограничения $\operatorname{Hom}_{\widehat{K}}(D, \mathscr{A}) \rightarrow \operatorname{Hom}_{\widehat{K}}(B, \mathscr{A})$ - эпиморфизм.

ОПредЕЛЕнИЕ 2 . Пусть $(K, \tau)$ - сайт, $D, B$ - предпучки множеств на $K, B \subset D, \Gamma: \mathscr{S}_{\tau} \rightarrow$ $\mathrm{Ab}$ зададим формулой $\Gamma(\mathscr{A})=\operatorname{ker}\left\{\operatorname{Hom}_{\widehat{K}}(D, \mathscr{A}) \rightarrow \operatorname{Hom}_{\widehat{K}}(B, \mathscr{A})\right\}=\{u: D \rightarrow \mathscr{A}|u| B=0\}$. Положим $H_{\tau}^{n}(D, B, \mathscr{A})=\left(R^{n} \Gamma\right)(\mathscr{A}), H_{\tau}^{n}(D, \mathscr{A})=H_{\tau}^{n}(D, \varnothing, \mathscr{A})$.

Teорема 1. Пусть $(K, \tau)-$ caüm. 1. Для любъх $D \in \mathrm{Ob}(\widehat{K}), B \in K_{D}, \forall \mathscr{A} \in \mathscr{S}_{\tau}$ имеется точная последовательность когомологий

$$
\begin{aligned}
0 \rightarrow \Gamma(\mathscr{A}) & \rightarrow \operatorname{Hom}_{\widehat{K}}(D, \mathscr{A}) \rightarrow \operatorname{Hom}_{\widehat{K}}(B, \mathscr{A}) \rightarrow H_{\tau}^{1}(D, B, \mathscr{A}) \rightarrow \cdots \\
\cdots & \rightarrow H_{\tau}^{n}(D, B, \mathscr{A}) \rightarrow H_{\tau}^{n}(D, \mathscr{A}) \rightarrow H_{\tau}^{n}(B, \mathscr{A}) \rightarrow H_{\tau}^{n+1}(D, B, \mathscr{A}) \rightarrow \cdots .
\end{aligned}
$$

2. Пусть $\mathscr{U}$ - некоторая совокупность пар $(E, F)$ предпучков множеств на $K$, таких, что $F \subset E$. Предположим, что для любого $\mathscr{U}$-вялого $\tau$-пучка $\mathscr{A}$ и для любой пары $(D, B) \in \mathscr{U}, H_{\tau}^{k}(D, \mathscr{A})=H_{\tau}^{k}(B, \mathscr{A})=0$ при $k>0$. Тогда следующие условия на абелев $\tau$-пучок $\mathscr{A}$ и число $n \geqslant 0$ әквивалентны:

(а) $\mathscr{A}$ обладает $\mathscr{U}$-вялой резольвентой длинь $n$;

(b) для любой резольвенты $0 \rightarrow \mathscr{A} \rightarrow \mathscr{B}^{0} \rightarrow \cdots \rightarrow \mathscr{B}^{n} \rightarrow 0$ пучка $\mathscr{A}$ длины $n$ из того, ито $\mathscr{B}^{i}-\mathscr{U}$-вялье $(0 \leqslant i \leqslant n-1)$, следует, ито $\mathscr{B}^{n}-\mathscr{U}$-вял;

(c) $\forall(D, B) \in \mathscr{U}, H_{\tau}^{n+1}(D, B, \mathscr{A})=0$;

(d) $\forall(D, B) \in \mathscr{U}, H_{\tau}^{k}(D, B, \mathscr{A})=0$ npu $k>n$;

(e) $\forall(D, B) \in \mathscr{U}, H_{\tau}^{n}(D, \mathscr{A}) \rightarrow H_{\tau}^{n}(B, \mathscr{A})-$ эпиморфизм;

(f) $\forall(D, B) \in \mathscr{U}, H_{\tau}^{n+1}(D, B, \mathscr{A}) \rightarrow H_{\tau}^{n+1}(D, \mathscr{A})$ - мономорфизм.

В частности, при $n=0$ получаеся когомологический критерий $\mathscr{U}$-вялости.

Таким образом, следуя [7] и [8], можно определить, например, $(\mathscr{U}, \mathscr{A})$-размерность Бредона предпучка множеств $E$, как минимальную длину $\mathscr{U}$-вялой резолшвенты $\mathscr{A}$, если $\mathscr{U}$ состоит из пар подпредпучков $E$. Из резулттатов автора [6] легко выводится для Тихоновских пространств обычное неравенство, связьвающее когомологическую размерность и размерность Бредона паракомпактных пространств. Кроме того, обычная когомологическая размерность топологического пространства может быть естественно определена, как размерность Бредона в подходящем сайте. 
Выделим условия, обеспечивающие ацикличность вялых пучков, необходимую, в частности, для применения предыдущей теоремы.

ОПРЕДЕЛЕНИЕ 3 . Пусть $(K, \tau)$ сайт, $D \in \mathrm{Ob}(\widehat{K}), P \subset K_{D, \tau} \cdot \beta=\left\{B_{i} \in K_{D} \mid i \in I\right\}$ называется ограниченно $P$ - $\tau$-представимым (или принадлежит классу $\mathscr{R}_{P}$ ), если $I$ можно так вполне упорядочить, что $\forall i>0\left[\cup\left\{B_{i} \cap B_{j} \mid j<i\right\}\right]_{\tau} \in P$. Если $K$ - ч.у.м., то $\alpha=\left\{a_{i} \in K \mid\right.$ $i \in I\} \in \mathscr{R}_{P} \Leftrightarrow j(\alpha)=\left\{j\left(a_{i}\right) \in K_{1} \mid i \in I\right\} \in \mathscr{R}_{P}$; если $\forall i, j \in I$ существует $a_{i} \wedge a_{j}$, то последнее равносильно следующему: $\forall i>0,\left\{a_{i} \wedge a_{j} \mid j<i\right\} \in \tau\left(C_{i}\right)$, где $C_{i} \in P$. Будем писать $\beta \in \mathscr{R}_{P}(B)$, если $\beta \in \mathscr{R}_{P}$ и $\beta \in \tau(B)$.

Нетрудно видеть, что если $Q \wedge L \subset P$, и для всякого $J \subset I$ мощности меньшей, чем $I,\left[\bigcup\left\{B_{i} \mid\right.\right.$ $i \in J\}]_{\tau} \in L,\left[B_{i}\right]_{\tau} \in Q$, то $\beta \in \mathscr{R}_{P}$. В частности, если $P$ подрешетка $K_{D, \tau}, I$ не более, чем счетно, $\left[B_{i}\right]_{\tau} \in P$, то $\beta \in \mathscr{R}_{P}$.

Пусть $K, L$ - множества, $\alpha=\left\{a_{i} \in K \mid i \in I\right\}, b \in K$. Через $\alpha \cup b$ будем обозначать семейство $\beta=\left\{a_{i}, b \mid i \in I\right\}$, причем если множество $I$ упорядоченно, то множеством индексов $\beta$ будем считать $I \cup\{-1\}$, где $-1<i \forall i \in I ; \alpha \subset L$ означает $a_{i} \in L \forall i \in I$.

Teopema 2. Пусть $(K, \tau)$ caüm, $K-$ ч.y.м., $D \in \mathrm{Ob}(\widehat{K}), L, M \subset K_{D, \tau}, \mathscr{U}=\{(A, B) \mid$ $A \in M, B \in L, B \subset A\}$, и дана точная последовательность (*) абелевых $\tau$-пучков на $K$ : $0 \rightarrow \mathscr{A} \stackrel{u}{\longrightarrow} \mathscr{B} \stackrel{v}{\longrightarrow} \mathscr{C} \rightarrow 0$.

1. Если $E \in K_{D}$, семейства $\beta \in \mathscr{R}_{L}(E)$, вписаннье в $M$ образуют конфинальную систему $\tau$-покрытий $E$, и в последовательности (*) $\mathscr{A}$ - $U$-вялый $\tau$-пучок, то последовательность $0 \rightarrow \operatorname{Hom}_{\widehat{K}}(E, \mathscr{A}) \rightarrow \operatorname{Hom}_{\widehat{K}}(E, \mathscr{B}) \rightarrow \operatorname{Hom}_{\widehat{K}}(E, \mathscr{C}) \rightarrow 0$ точна.

2. Пусть для каждого $H \in L$, вписанного в $M$, семейства $\beta \in \mathscr{R}_{L}(H)$, вписанные в $M$, образуют конфинальную систему $\tau$-покрытий $H$. Тогда

a) $Е с л и E \in K_{D}$ и семейства класса $\mathscr{R}_{L}(E)$, вписаннье в $M$, образуют конфинальную систему $\tau$-покрытий $E$, то для любого $\mathscr{U}$-вялого $\tau$-пучка $\mathscr{D}, H_{\tau}^{n}(E, \mathscr{D})=0$ $\forall n \geqslant 1$. В частности, так будет, если имеется $\beta=\left\{B_{i} \mid i \in I\right\} \in \mathscr{R}_{L}(E), \beta$ вписано в $M,\left[B_{i}\right]_{\tau} \in L$, и выполняется соотношение $L \wedge(L \vee L) \subset L$.

b) Если в последовательности (*) пучки $\mathscr{A}$ и $\mathscr{B}-\mathscr{U}$-вялье, то и $\mathscr{C}-\mathscr{U}$-вял.

3. Пусть $E, F \in K_{D}, F \subset E$.

а) Если существует семейство а подпредпучков $E$, вписанное в $M$, такое, что $[\alpha \cap F]_{\tau} \subset L, u \alpha \cup F \in \mathscr{R}_{L}(E)$, то для всякого $\mathscr{U}$-вялого пучка $\mathscr{A}$ отображсение $\operatorname{Hom}_{\widehat{K}}(E, \mathscr{A}) \rightarrow \operatorname{Hom}_{\widehat{K}}(F, \mathscr{A})$ является эпимор физмом.

b) Если в каждое $\tau$-покрытие $E$ вписывается такое семейство $\alpha \prec M$ подпредпучков $D$, что $\alpha \cup F \in \mathscr{R}_{L}(E)$, то из того, что пучок $\mathscr{A}$ в последовательносmи (*) является $\mathscr{U}$-вяльм , следует, ито $\forall s \in \operatorname{Hom}_{\widehat{K}}(E, \mathscr{C}), \forall t \in \operatorname{Hom}_{\widehat{K}}(F, \mathscr{B})$, таких, что $v \circ t=s \mid F$, имеется такой гомоморфизм $\tilde{t}: E \rightarrow \mathscr{B}$, что $\tilde{t} \mid F=t$, $v \circ \tilde{t}=s$

\section{СПИСОК ЛИТЕРАТУРЫ}

[1] Годеман Р. Гомологическая алгебра и теория пучков. М.: ИЛ, 1961. [2] Скурихин Е. Е. // Матем. весник. Белград. 1987. Т. 39. С. 65-75, 213-224. [3] Grothendieck A. (with Artin M., Verdier J.-L.) // Lect. Note in Math.. V. 269, 270. P. 1972-1973. [4] Скурихин Е.Е. // Труды МИРАН. 1992. Т. 193. С. 169-173. [5] Ситников К. А., Скурихин Е. Е. // Труды МИАН. 1991. Т. 196. С. 156-160. [6] Скурихин Е. Е. Пучковые когомологии и полные брауэровы решетки. Владивосток: Дальнаука, 1993. [7] Bredon G. E. Sheaf Theory. New York, 1967. [8] Кузьминов В. И., Шведов И. А. // Докл. АН СССР. 1976. T. 231. № 1. C. 24-27. 